trust funds at the disposal of the University. As is usual, the provision of a sufficient revenue is left to luck. If the amount of research is to be enough to justify the accommodation, much moro than is at present forthcoming will be required to provide modest salaries for the upkeep of research workers. 'There is no lack of suitable graduates in Australia who are anxious to devote themselves to investigation if they can see a reasonable prospect of a living wage. At present they come to Great Britain with Rhodes and Beit and other research studentships and when trained remain, as there are not openings for them in their mother country. This drainage is not too good for Australia.

Both University and Hospital are to be congratulated on having secured convenient modern accommodation and equipment for teaching and research in pathology and for the better treatment of patients and investigation of their diseases. They are still more to be congratulated on having surmounted the difficulties always attending combined action by the two corporate bodies under separate governance.

Close association of pathology with clinical medicine is desirable. A pathological institute can function apart from a hospital, but its scope is restricted, and researches tend to become remote from the immediate problems of human medicine and the workers lack the stimulus to effort which is afforded by contact with the sick.

An unusual feature in the new Institute at Adelaide is the inclusion of the study of animal pathology as one of its main objects. For some years investigations of diseases of farm animals have been carried on at the pathological laboratory at the Adelaide Hospital, the Government providing a veterinary officer and technical staff for the purpose. The continuation of this association of the study of human and animal diseases will be both convenient and advantageous, for pathology has no specific boundaries, and have not most of the recent discoveries in human pathology been, perforce, achieved by experimenting with animals?

\title{
SYNTHETIC SOUND
}

$\mathrm{I}_{\mathrm{b}}^{\mathrm{N}}$ Philips Technical Review of June, which is edited by the Research Laboratory of Philips Glow Lamp Factory, Eindhoven, Holland, there is an interesting paper on "Synthetic Sound" by J. F. Schouten. A sound of prescribed character of periodic vibra. tions, in other words, a sound consisting of harmonic components of prescribed amplitude and phase, is obtained synthetically. This method permits a closer study of a number of problems which are connected with the nature of the perception of sound. The principle used is to make a beam of light vary as a function of the time in the desired wave form. The beam is incident on a photo-electric cell and actuates a loud-speaker. A paper stencil is made and is uniformly illuminated by a point source of light from a tungsten are lamp placed at a considerable distance away. Behind the stencil there is a rotating aluminium disk in which nine slits have been made, each with a width of $1 \mathrm{~mm}$. and at distances of $40^{\circ}$ from each other. Behind the disk, which is driven by a motor, is a lens which focuses the light source upon the photo-electric cell. The only light which can fall upon the photo-electric cell is that which has passed through the part of the stencil cut away and one of the slits. When the disk is turning, the amount of light transmitted at each moment is proportional to the height of the part cut away at the point behind which there is a slit at that moment.

The centre of the wave form is situated on the extension of the axle of the motor. With the arrange. ment used negative values of the light transmissibility cannot be produced, and so a constant quantity equal to the largest negative value occurring is added to all the ordinates of the wave form to be reproduced. The frequency was determined by regulating the number of revolutions per minute of the motor. If the desired form is given as the sum of a number of sine-shaped components, the components can be added and the resultant wave form cut out. It is easy to cut out the forms separately one above the other.
An attractive property of this apparatus is that it makes it possible to study directly the influence exerted on sound perception by changes in the wave form. For examplo, when several components havo been cut out one above the other, any one of these components can be made to disappear, simply by screening this part of the stencil from the light.

According to a law of acoustics which was formu. lated by Ohm, a definite pure tone will bo observed in a synthetic sound, when a component of the frequency in question occurs in the Fourier analysis of the wave form. According to a rule enunciated by Helmholtz, the sound perception will further depend entirely on the relative intensity with which the different components occur and will be independent of the relative phase of these components.

The author accounts for these facts in a simplo way, which seems to be permissible anatomically, that there are in the ear a large number of resonators tuned for different frequencies. This mechanism explains in the first place the fact that the ear carries out, as it were, a.Fourier analysis of the sound while it furthermore makes it possible to suppose that the stimuli which are sent from each resonator to the brain depend exclusively upon the intensity, and not upon the phase of the component in question. The author shows how to test this rule by his apparatus.

Four wave forms were built up of very different shape but all having the same harmonics from $I$ to 20. It was found that these four totally different wave forms were quite indistinguishable as to their sound impression. It is also stated that non-linear distortion occurs in tho ear. This is manifested by the fact that when a pure tone of sufficient intensity is heard, higher harmonics are formed in the ear; when two tones of different frequency are heard at the same time, new tones are formed with frequencies which are linear combinations of the two tones heard (combination tones). The most obvious combination tone is the difference tone and this is the one that was first discovered. 